\title{
Dynamics analysis for a discrete dynamic competition model
}

\author{
Xiuqin Yang ${ }^{1}$, Feng Liu ${ }^{2 *}$ (D), Qingyi Wang ${ }^{2}$ and Hua O. Wang ${ }^{3}$
}

"Correspondence: Ifpost@163.com ${ }^{2}$ School of Automation, China University of Geosciences, Wuhan, P.R. China

Full list of author information is available at the end of the article

\begin{abstract}
In this paper, the dynamics of a discrete market share attraction model are investigated. It shows that the system can undergo flip bifurcation and chaos. The stability and bifurcation of a market share attraction model are analyzed by using the bifurcation theory and the center manifold theorem. The system displays complex dynamical behaviors, including period-1, 2, 4, 6, 8, 16 orbits, invariant cycle, a cascade of period-doubling, quasi-periodic orbits, and the chaotic sets. Numerical simulations illustrate the analysis and results.
\end{abstract}

Keywords: Stability; Flip bifurcation; Discrete; Dynamic competition model

\section{Introduction}

Over the years, dynamics analysis of economic systems has mainly focused on the stability behavior of the system equilibrium point. According to the theory of nonlinear dynamical systems, the phenomena of fluctuations over time are not stochastic influences arising from external factors, but because of the nonlinear relationship between the variables of the economic system. Market share attraction models are used for analyzing Interbrand competitive structures. These models have received more and more attention [1-5].

Recently, many research papers suggested that the mathematical model of economic system dynamics is more realistic and appropriate when it is modeled by discrete-time equations. The dynamics of the discrete-time models can exhibit much richer dynamics than those observed in continuous-time counterparts and can lead to chaotic behaviors [6-20].

A market share attraction model is as follows:

$$
\left\{\begin{array}{l}
x_{t+1}=x_{t}+\lambda_{1} x_{t}\left(B s_{1 t}-x_{t}\right), \\
y_{t+1}=y_{t}+\lambda_{2} y_{t}\left(B s_{2 t}-y_{t}\right)
\end{array}\right.
$$

where $x_{t}$ and $y_{t}$ denote the marketing efforts of the two brands respectively. $B$ denotes the total sales potential of the market. $s_{1 t}=x_{t}^{\beta_{1}} /\left(x_{t}^{\beta_{1}}+k y_{t}^{\beta_{2}}\right), s_{2 t}=k y_{t}^{\beta_{2}} /\left(x_{t}^{\beta_{1}}+k y_{t}^{\beta_{2}}\right)$. The parameters $\beta_{1}$ and $\beta_{2}$ denote the elasticities of the attraction of firm (or brand) $i$ with regard to the effort of firm $i$. The parameter $k$ denotes the relative effectiveness ratio of the effort made by the firms. The parameters $\lambda_{1}$ and $\lambda_{2}$ measure the rate of adjustment.

(c) The Author(s) 2019. This article is distributed under the terms of the Creative Commons Attribution 4.0 International License (http://creativecommons.org/licenses/by/4.0/), which permits unrestricted use, distribution, and reproduction in any medium, provided you give appropriate credit to the original author(s) and the source, provide a link to the Creative Commons license, and indicate if changes were made. 
From system (1.1), the mapping form is obtained:

$$
\left\{\begin{array}{l}
x^{\prime} \rightarrow x+\lambda_{1} x\left(B \frac{x^{\beta_{1}}}{x^{\beta_{1}}+k y^{\beta_{2}}}-x\right) \\
y^{\prime} \rightarrow y+\lambda_{2} y\left(B \frac{k y_{2}}{x^{\beta_{1}+k y^{\beta_{2}}}}-y\right)
\end{array}\right.
$$

where $\lambda_{1}, \lambda_{2}, \beta_{1}, \beta_{2}, k$, and $B$ are real and positive parameters. We consider only the values of the exponents $\beta_{1}$ and $\beta_{2}$ at the interval $(0,1)$ since empirical studies show that realistic values are in this range.

Our objectives are to study the dynamical behaviors of system (1.2). Sufficient conditions for the existence of flip bifurcation are derived by using the bifurcation theory and the center manifold theorem. Moreover, system (1.2) shows a rich variety of nonlinear dynamics, including bifurcations and chaos.

The paper is organized as follows. In Sect. 2, the stability and existence of the fixed points of system (1.2) are discussed. In Sect. 3, the existence of flip bifurcation is obtained by using the center manifold theorem and bifurcation theory. Numerical simulations are illustrated to confirm the theoretical results in Sect. 4. Some conclusions are presented in Sect. 5 .

\section{The existence and stability of the fixed points}

We study the existence of fixed points. Also, we investigate the stability properties of system (1.2). The fixed points of map (1.2) are the solutions of the following equations:

$$
\left\{\begin{array}{l}
\lambda_{1} x\left(B \frac{x^{\beta_{1}}}{x^{\beta_{1}}+k y^{\beta_{2}}}-x\right)=0, \\
\lambda_{2} y\left(B \frac{k y^{\beta_{2}}}{x^{\beta_{1}+k y^{\beta_{2}}}}-y\right)=0 .
\end{array}\right.
$$

For all parameter values, equation (2.1) has three solutions $O(0,0), P_{1}(B, 0)$, and $P_{2}(0, B)$. As the map does not define $O$, it is not a fixed point. $P_{1}$ and $P_{2}$ are the fixed points of the map.

There is an interior fixed point $E(x, y)$ of map (1.2), which is the solution of the following system:

$$
\left\{\begin{array}{l}
B \frac{x^{\beta_{1}}}{x^{\beta_{1}+k y^{\beta_{2}}}}-x=0 \\
B \frac{k \beta^{\beta_{2}}}{x^{\beta_{1}+k y^{\beta_{2}}}}-y=0
\end{array}\right.
$$

From equation (2.2), we have

$$
G(x)=k^{1 /\left(1-\beta_{2}\right)} x^{\left(1-\beta_{1}\right) /\left(1-\beta_{2}\right)}+x-B=0 .
$$

$G$ is a continuous function, $G(B)>0, G(0)<0$, and $G^{\prime}(x)>0$ for $x>0$, so there is a unique positive solution, $x^{*} \in(0, B)$, the fixed point is $E\left(x^{*}, B-x^{*}\right)$.

A particularly simple solution is obtained in the case $\beta_{1}=\beta_{2}, x^{*}=B /\left(1+k^{1 /\left(1-\beta_{2}\right)}\right)$.

We will study the local stability of the fixed points.

The Jacobian matrix of system $(1.2)$ at $(x, y)$ is given as follows:

$$
J(x, y)=\left[\begin{array}{cc}
1+\lambda_{1} a_{1} & -\lambda_{1} b_{1} \\
-\lambda_{2} a_{2} & 1+\lambda_{2} b_{2}
\end{array}\right],
$$


where

$$
\begin{aligned}
& a_{1}=\left(\frac{B x^{2 \beta_{1}}+B k\left(\beta_{1}+1\right) x^{\beta_{1}} y^{\beta_{2}}}{\left(x^{\beta_{1}}+k y^{\beta_{2}}\right)^{2}}-2 x\right), \\
& b_{1}=\frac{B k \beta_{2} y^{\beta_{2}-1} x^{\beta_{1}+1}}{\left(x^{\beta_{1}}+k y^{\beta_{2}}\right)^{2}}, \\
& a_{2}=\frac{B k \beta_{1} y^{\beta_{2}+1} x^{\beta_{1}-1}}{\left(x^{\beta_{1}}+k y^{\beta_{2}}\right)^{2}}, \\
& b_{2}=\left(\frac{B k^{2} y^{2 \beta_{2}}+B k\left(\beta_{2}+1\right) x^{\beta_{1}} y^{\beta_{2}}}{\left(x^{\beta_{1}}+k y^{\beta_{2}}\right)^{2}}-2 y\right) .
\end{aligned}
$$

So the characteristic equation of the Jacobian matrix $J$ can be written as

$$
s^{2}-\left(2+\lambda_{1} a_{1}+\lambda_{2} b_{2}\right) s+\left(1+\lambda_{1} a_{1}+\lambda_{2} b_{2}+\lambda_{1} \lambda_{2}\left(a_{1} b_{2}-a_{2} b_{1}\right)\right)=0 .
$$

In order to study the stability at the positive fixed point, we use the following lemmas, which can be easily proved by the relations between roots and coefficients of the quadratic equation.

Let $F(s)=s^{2}+M s+N$ be the characteristic equation of eigenvalues associated with the Jacobian matrix evaluated at a fixed point $\left(x^{*}, y^{*}\right)$. Let $s_{1}$ and $s_{2}$ be the two roots of $F(s), M$ and $N$ be coefficients of the quadratic equation.

Lemma 2.1 ([21]) We have the following definitions for $\left(x^{*}, y^{*}\right)$ :

(1) $\left(x^{*}, y^{*}\right)$ is called a sink if $\left|s_{1}\right|<1$ and $\left|s_{2}\right|<1$, so the sink is locally asymptotically stable;

(2) $\left(x^{*}, y^{*}\right)$ is called a source if $\left|s_{1}\right|>1$ and $\left|s_{2}\right|>1$, so the source is locally unstable;

(3) $\left(x^{*}, y^{*}\right)$ is called a saddle if $\left|s_{1}\right|>1$ and $\left|s_{2}\right|<1$ (or $\left|s_{1}\right|<1$ and $\left.\left|s_{2}\right|>1\right)$;

(4) $\left(x^{*}, y^{*}\right)$ is non-hyperbolic if either $\left|s_{1}\right|=1$ or $\left|s_{2}\right|=1$.

Lemma 2.2 ([21]) Let $F(s)=s^{2}+M s+N$. Suppose that $F(1)>0, s_{1}$ and $s_{2}$ are two roots of $F(s)=0$. Then

(1) $\left|s_{1}\right|<1$ and $\left|s_{2}\right|<1$ if and only if $F(-1)<0, N<1$;

(2) $\left|s_{1}\right|<1$ and $\left|s_{2}\right|>1$ (or $\left|s_{1}\right|>1$ and $\left|s_{2}\right|<1$ ) if and only if $F(-1)<0$;

(3) $\left|s_{1}\right|>1$ and $\left|s_{2}\right|>1$ if and only if $F(1)>0, N>1$;

(4) $s_{1}=-1$ and $\left|s_{2}\right| \neq 1$ if and only if $F(-1)=0$ and $M \neq 0,2$;

(5) $s_{1}$ and $s_{2}$ are complex and $\left|s_{1}\right|=\left|s_{2}\right|=1$ if and only if $M^{2}-4 N<0$ and $N=1$.

Now we state the following three propositions.

Proposition 1 The eigenvalues of $J(B, 0)$ are $s_{1}=1-\lambda_{1} B$ and $s_{2}=1$, then $(B, 0)$ is nonhyperbolic.

Proposition 2 The eigenvalues of $J(0, B)$ are $s_{1}=1$ and $s_{2}=1-\lambda_{1} B$, then $(0, B)$ is nonhyperbolic.

For the fixed point $O(0,0)$, we can get a solution in the case of identical firms. 
Here we consider the symmetric case of identical firms obtained for

$$
\lambda_{1}=\lambda_{2}=\lambda>0, \quad \beta_{1}=\beta_{2}=\beta>0, \quad k=1 .
$$

Note that this steady state allocation belongs to the diagonal $\Delta=\{(x, y) \mid x=y\}$.

For the symmetric map, the Jacobian matrix, computed at a point of the diagonal $\Delta$, is

$$
J(x, x)=\left[\begin{array}{cc}
1-2 \lambda x+\frac{\lambda B(\beta+2)}{4} & -\frac{\lambda B \beta}{4} \\
-\frac{\lambda B \beta}{4} & 1-2 \lambda x+\frac{\lambda B(\beta+2)}{4}
\end{array}\right] .
$$

The eigenvalues are

$$
s_{1}=1+\frac{1}{2} \lambda B-2 \lambda x, \quad s_{2}=1+\frac{1}{2} \lambda B(1+\beta)-2 \lambda x .
$$

The fixed point $O(0,0)$ has the following topological properties:

$$
s_{1}=1+\frac{1}{2} \lambda B, \quad s_{2}=1+\frac{1}{2} \lambda B(1+\beta) .
$$

Proposition 3 The eigenvalues of $J(0,0)$ are $s_{1}=1+\frac{1}{2} \lambda B>1$ and $s_{2}=1+\frac{1}{2} \lambda B(1+\beta)>1$, then $(0,0)$ is a source; the source is locally unstable.

For the fixed point $E\left(x^{*}, B-x^{*}\right), x^{*} \in(0, B)$, we can get a special solution in the case of identical firms $E\left(\frac{B}{2}, \frac{B}{2}\right)$.

Here we consider the symmetric case of identical firms obtained for

$$
\lambda_{1}=\lambda_{2}=\lambda, \quad \beta_{1}=\beta_{2}=\beta .
$$

$J(x, y)$ evaluated at the interior fixed point

$$
J(x, y)=\left[\begin{array}{cc}
1+\lambda a_{1} & -\lambda b_{1} \\
-\lambda a_{2} & 1+\lambda b_{2}
\end{array}\right],
$$

where

$$
\begin{aligned}
& a_{1}=\left(\frac{B x^{2 \beta}+B k(\beta+1) x^{\beta} y^{\beta}}{\left(x^{\beta}+k y^{\beta}\right)^{2}}-2 x\right), \quad b_{1}=\frac{B k \beta y^{\beta-1} x^{\beta+1}}{\left(x^{\beta}+k y^{\beta}\right)^{2}}, \\
& a_{2}=\frac{B k \beta y^{\beta+1} x^{\beta-1}}{\left(x^{\beta}+k y^{\beta}\right)^{2}}, \quad b_{2}=\left(\frac{B k^{2} y^{2 \beta}+B k(\beta+1) x^{\beta} y^{\beta}}{\left(x^{\beta}+k y^{\beta}\right)^{2}}-2 y\right) .
\end{aligned}
$$

The characteristic equation of (2.7) evaluated at the positive fixed point $E\left(x^{*}, y^{*}\right)$ can be written as

$$
s^{2}-(2+G \lambda) s+\left(1+G \lambda+H \lambda^{2}\right)=0,
$$

where $G=a_{1}+b_{2}, H=a_{1} b_{2}-a_{2} b_{1}$.

Let $F(s)=s^{2}-(2+G \lambda) s+\left(1+G \lambda+H \lambda^{2}\right)$.

Then $F(1)=H \lambda^{2}, F(-1)=H \lambda^{2}+2 G \lambda+4$.

Using Lemma 2.1, we obtain the local dynamics of the fixed point $E\left(x^{*}, y^{*}\right)$. 
Proposition 4 Let $E\left(x^{*}, y^{*}\right)$ be the positive fixed point of Eq. (1.2);

1. $E$ is a sink if one of the following conditions holds:

(a) $-2 \sqrt{H} \leq G<0$ and $0<\lambda<-\frac{G}{H}$.

(b) $G<-2 \sqrt{H}$ and $0<\lambda<\frac{-G-\sqrt{G^{2}-4 H}}{H}$.

So E locally asymptotically stable.

2. $E$ is a source if one of the following conditions holds:

(a) $-2 \sqrt{H} \leq G<0$ and $\lambda>-\frac{G}{H}$.

(b) $G<-2 \sqrt{H}$ and $\lambda>\frac{-G+\sqrt{G^{2}-4 H}}{H}$.

(c) $G \geq 0$.

3. $E$ is a saddle if the following condition holds:

$$
G<-2 \sqrt{H} \text { and } \frac{-G-\sqrt{G^{2}-4 H}}{H}<\lambda<\frac{-G+\sqrt{G^{2}-4 H}}{H} \text {. }
$$

4. $E$ is non-hyperbolic if one of the following conditions holds:

(a) $G<-2 \sqrt{H}$ and $\lambda=\frac{-G \pm \sqrt{G^{2}-4 H}}{H}$ and $\lambda \neq-\frac{2}{G},-\frac{4}{G}$.

(b) $-2 \sqrt{H}<G<0$ and $\lambda=-\frac{G}{H}$.

\section{Bifurcation analysis}

In this section, we discuss the flip bifurcation in system (1.2) at the positive fixed point $E\left(x^{*}, y^{*}\right)$. We choose parameter $\lambda$ as a bifurcation parameter to study the flip bifurcation of $E\left(x^{*}, y^{*}\right)$ by using the center manifold theorem and the bifurcation theory [22-25].

Let $F_{B 1}=\left\{(B, k, \beta, \lambda): \lambda=\frac{-G+\sqrt{G^{2}-4 H}}{H}, G<-2 \sqrt{H}, B, k, \beta, \lambda>0\right\}$, or $F_{B 2}=\{(B, k, \beta, \lambda): \lambda=$ $\left.\frac{-G-\sqrt{G^{2}-4 H}}{H}, G<-2 \sqrt{H}, B, k, \beta, \lambda>0\right\}$.

Let $H_{B}=\left\{(B, k, \beta, \lambda): \lambda=-\frac{G}{H},-2 \sqrt{H}<G<0, B, k, \beta, \lambda>0\right\}$.

The fixed point $\left(x^{*}, y^{*}\right)$ can undergo a flip bifurcation when parameters vary in a small neighborhood of $F_{B 1}$ or $F_{B 2}$, and the Neimark-Sacker bifurcation of $E\left(x^{*}, y^{*}\right)$ if parameters vary in a small neighborhood of $H_{B}$.

\subsection{Flip bifurcation analysis}

We will discuss the flip bifurcation of (1.2) at $E\left(x^{*}, y^{*}\right)$ when parameters vary in the small neighborhood of $F_{B 1}$. Similar arguments can be applied to the other case $F_{B 2}$. Taking parameters $\left(B, k, \beta, \lambda_{1}\right)$ arbitrarily from $F_{B 1}$, we consider system (1.2) with $\left(B, k, \beta, \lambda_{1}\right)$, which is described by

$$
\left\{\begin{array}{l}
x^{\prime} \rightarrow x+\lambda_{1} x\left(B \frac{x^{\beta}}{x^{\beta}+k y^{\beta}}-x\right), \\
y^{\prime} \rightarrow y+\lambda_{1} y\left(B \frac{k y^{\beta}}{x^{\beta}+k y^{\beta}}-y\right) .
\end{array}\right.
$$

Map (3.1) has a unique positive fixed point $E\left(x^{*}, y^{*}\right)$, whose eigenvalues are $s_{1}=-1, s_{2}=$ $3+G \lambda_{1}$ with $\left|s_{2}\right| \neq 1$ by Proposition 4 , where $x^{*}=B /\left(1+k^{1 /(1-\beta)}\right), y^{*}=B-\left[B /\left(1+k^{1 /(1-\beta)}\right)\right]$.

Choosing $\lambda^{*}$ as a bifurcation parameter, we consider a perturbation of (3.1) as follows:

$$
\left\{\begin{array}{l}
x^{\prime} \rightarrow x+\left(\lambda_{1}+\lambda^{*}\right) x\left(B \frac{x^{\beta}}{x^{\beta}+k y^{\beta}}-x\right), \\
y^{\prime} \rightarrow y+\left(\lambda_{1}+\lambda^{*}\right) y\left(B \frac{k y^{\beta}}{x^{\beta}+k y^{\beta}}-y\right),
\end{array}\right.
$$

where $\left|\lambda^{*}\right| \ll 1$, which is a small perturbation parameter. 
Let $u=x-x^{*}$ and $v=y-y^{*}$. Then we transform the fixed point $E\left(x^{*}, y^{*}\right)$ of map (3.2) into the origin. We have

$$
\begin{aligned}
& \left(\begin{array}{l}
u \\
v
\end{array}\right) \\
& \rightarrow\left(\begin{array}{c}
a_{11} u+a_{12} v+a_{13} u^{2}+a_{14} u v+a_{15} v^{2}+e_{1} u^{3}+e_{2} u^{2} v+e_{3} v^{2} u+e_{4} v^{3} \\
+b_{1} u \lambda^{*}+b_{2} v \lambda^{*}+b_{3} u^{2} \lambda^{*}+b_{4} u v \lambda^{*}+b_{5} v^{2} \lambda^{*}+O\left(\left(|u|+|v|+\left|\lambda^{*}\right|\right)^{4}\right) \\
a_{21} u+a_{22} v+a_{23} u^{2}+a_{24} u v+a_{25} v^{2}+d_{1} u^{3}+d_{2} u^{2} v+d_{3} v^{2} u+d_{4} v^{3} \\
+c_{1} u \lambda^{*}+c_{2} v \lambda^{*}+c_{3} u^{2} \lambda^{*}+c_{4} u v \lambda^{*}+c_{5} v^{2} \lambda^{*}+O\left(\left(|u|+|v|+\left|\lambda^{*}\right|\right)^{4}\right)
\end{array}\right),
\end{aligned}
$$

where

$$
\begin{aligned}
& a_{11}=1+\lambda_{1}\left(\frac{B x^{* 2 \beta}+B k(\beta+1) x^{* \beta} y^{* \beta}}{\left(x^{* \beta}+k y^{* \beta}\right)^{2}}-2 x^{*}\right), \quad a_{12}=-\frac{\lambda_{1} B k \beta y^{* \beta-1} x^{* \beta+1}}{\left(x^{* \beta}+k y^{* \beta}\right)^{2}}, \\
& a_{13}=\frac{\lambda_{1} B k \beta x^{* \beta-1} y^{* \beta}\left[(1-\beta) x^{* \beta}+k(1+\beta) y^{* \beta}\right]}{\left(x^{* \beta}+k y^{* \beta}\right)^{3}}-2 \text {, } \\
& a_{14}=\lambda_{1} k B \beta x^{* \beta} y^{* \beta-1} \frac{(\beta-1) x^{* \beta}-k(\beta+1) y^{* \beta}}{\left(x^{* \beta}+k y^{* \beta}\right)^{3}} \text {, } \\
& a_{15}=\lambda_{1} k B \beta y^{* \beta-2} x^{* \beta+1} \frac{(1-\beta) x^{* \beta}+k(\beta+1) y^{* \beta}}{\left(x^{* \beta}+k y^{* \beta}\right)^{3}} \text {, } \\
& b_{1}=\left(\frac{B x^{* 2 \beta}+B k(\beta+1) x^{* \beta} y^{* \beta}}{\left(x^{* \beta}+k y^{* \beta}\right)^{2}}-2 x^{*}\right) \text {, } \\
& b_{2}=-\frac{B k \beta y^{* \beta-1} x^{* \beta+1}}{\left(x^{* \beta}+k y^{* \beta}\right)^{2}} \text {, } \\
& b_{3}=\frac{B k \beta x^{* \beta-1} y^{* \beta}\left[(1-\beta) x^{* \beta}+k(1+\beta) y^{* \beta}\right]}{\left(x^{* \beta}+k y^{* \beta}\right)^{3}}-2 \text {, } \\
& b_{4}=k B \beta x^{* \beta} y^{* \beta-1} \frac{(\beta-1) x^{* \beta}-k(\beta+1) y^{* \beta}}{\left(x^{* \beta}+k y^{* \beta}\right)^{3}}, \\
& b_{5}=k B \beta y^{* \beta-2} x^{* \beta+1} \frac{(1-\beta) x^{* \beta}+(k \beta+1) y^{* \beta}}{\left(x^{* \beta}+k y^{* \beta}\right)^{3}} \text {, } \\
& a_{21}=-\frac{\lambda_{1} B k \beta y^{* \beta+1} x^{* \beta-1}}{\left(x^{* \beta}+k y^{* \beta}\right)^{2}} \\
& a_{22}=1+\lambda_{1}\left(\frac{B k^{2} y^{* 2 \beta}+B k(\beta+1) x^{* \beta} y^{* \beta}}{\left(x^{* \beta}+k y^{* \beta}\right)^{2}}-2 y^{*}\right) \text {, } \\
& a_{23}=\lambda_{1} k B \beta x^{* \beta-2} y^{* \beta+1} \frac{(1-\beta) k y^{* \beta}+(\beta+1) x^{* \beta}}{\left(x^{* \beta}+k y^{* \beta}\right)^{3}} \text {, } \\
& a_{24}=\lambda_{1} k B \beta x^{* \beta-2} y^{* \beta+1} \frac{(\beta-1) k y^{* \beta}-(\beta+1) x^{* \beta}}{\left(x^{* \beta}+k y^{* \beta}\right)^{3}} \text {, } \\
& a_{25}=\lambda_{1} k B \beta x^{* \beta} y^{* \beta-1} \frac{(1-\beta) k y^{* \beta}+(\beta+1) x^{* \beta}}{\left(x^{* \beta}+k y^{* \beta}\right)^{3}}-2 \text {, } \\
& c_{1}=-\frac{B k \beta y^{* \beta+1} x^{* \beta-1}}{\left(x^{* \beta}+k y^{* \beta}\right)^{2}},
\end{aligned}
$$




$$
\begin{aligned}
& c_{2}=\left(\frac{B k^{2} y^{* 2 \beta}+B k(\beta+1) x^{* \beta} y^{* \beta}}{\left(x^{* \beta}+k y^{* \beta}\right)^{2}}-2 y^{*}\right) \text {, } \\
& c_{3}=k B \beta x^{* \beta-2} y^{* \beta+1} \frac{(1-\beta) k y^{* \beta}+(\beta+1) x^{* \beta}}{\left(x^{* \beta}+k y^{* \beta}\right)^{3}} \text {, } \\
& c_{4}=k B \beta x^{* \beta-2} y^{* \beta+1} \frac{(\beta-1) k y^{* \beta}-(\beta+1) x^{* \beta}}{\left(x^{* \beta}+k y^{* \beta}\right)^{3}} \text {, } \\
& c_{5}=k B \beta x^{* \beta} y^{* \beta-1} \frac{(1-\beta) k y^{* \beta}+(\beta+1) x^{* \beta}}{\left(x^{* \beta}+k y^{* \beta}\right)^{3}}-2 \text {, } \\
& d_{1}=\frac{\lambda_{1} B k \beta y^{* \beta+1} x^{* \beta-3}\left[\left(-3 \beta-2-\beta^{2}\right) x^{* 2 \beta}+4 k\left(\beta^{2}-1\right) x^{* \beta} y^{* \beta}+k^{2}\left(3 \beta-\beta^{2}-2\right) y^{* 2 \beta}\right]}{\left(x^{* \beta}+k y^{* \beta}\right)^{4}}, \\
& d_{2}=\frac{\lambda_{1} B k \beta x^{* \beta-2} y^{* \beta}\left[k^{2}(\beta-1)^{2} y^{* 2 \beta}+(\beta+1)^{2} x^{* 2 \beta}+2 k\left(1-2 \beta^{2}\right) x^{* \beta} y^{* \beta}\right]}{\left(x^{* \beta}+k y^{* \beta}\right)^{4}}, \\
& d_{3}=\frac{\left.\lambda_{1} B k \beta^{2} x^{* \beta-1} y^{* \beta-1}\left[-(1+\beta) x^{* 2 \beta}+k^{2}(1-\beta) y^{* 2 \beta}\right)+4 k \beta x^{* \beta} y^{* \beta}\right]}{\left(x^{* \beta}+k y^{* \beta}\right)^{4}}, \\
& d_{4}=\frac{\lambda_{1} B k \beta y^{* \beta-2} x^{* \beta}\left[\left(\beta^{2}-1\right)\left(k^{2} y^{* 2 \beta}+x^{* 2 \beta}\right)-2 k\left(1+2 \beta^{2}\right) x^{* \beta} y^{* \beta}\right]}{\left(x^{* \beta}+k y^{* \beta}\right)^{4}} . \\
& e_{1}=\frac{\lambda_{1} B k \beta x^{* \beta-2} y^{* \beta}\left[\left(\beta^{2}-1\right)\left(x^{* 2 \beta}+k^{2} y^{* 2 \beta}\right)-2 k\left(1+2 \beta^{2}\right) x^{* \beta} y^{* \beta}\right]}{\left(x^{* \beta}+k y^{* \beta}\right)^{4}}, \\
& e_{2}=\frac{\left.\lambda_{1} B k \beta^{2} x^{* \beta-1} y^{* \beta-1}\left[(1-\beta) x^{* 2 \beta}-k^{2}(1+\beta) y^{* 2 \beta}\right)+4 k \beta x^{* \beta} y^{* \beta}\right]}{\left(x^{* \beta}+k y^{* \beta}\right)^{4}}, \\
& e_{3}=\frac{\lambda_{1} B k \beta x^{* \beta} y^{* \beta-2}\left[2\left(2 \beta-1-\beta^{2}\right) x^{* 2 \beta}-k\left(1+\beta^{2}\right) x^{* \beta} y^{* \beta}+k^{2}(\beta+1)^{2} y^{* 2 \beta}\right]}{\left(x^{* \beta}+k y^{* \beta}\right)^{4}} \text {, } \\
& e_{4}=\left(\lambda _ { 1 } B k \beta x ^ { * \beta + 1 } y ^ { * \beta - 3 } \left[\left(2 \beta-3-\beta^{2}\right) x^{* 2 \beta}+2 k\left(2 \beta^{2}-\beta-3\right) x^{* \beta} y^{* \beta}\right.\right. \\
& \left.\left.-k^{2}\left(\beta^{2}+4 \beta+3\right) y^{* 2 \beta}\right]\right) /\left(\left(x^{* \beta}+k y^{* \beta}\right)^{4}\right) \text {. }
\end{aligned}
$$

Constructing an invertible matrix

$$
T=\left(\begin{array}{cc}
a_{12} & a_{12} \\
-1-a_{11} & s_{2}-a_{11}
\end{array}\right)
$$

and using the translation

$$
\left(\begin{array}{l}
u \\
v
\end{array}\right)=T\left(\begin{array}{l}
\tilde{x} \\
\tilde{y}
\end{array}\right)
$$

then system (3.3) becomes

$$
\left(\begin{array}{l}
\tilde{x} \\
\tilde{y}
\end{array}\right) \rightarrow\left(\begin{array}{cc}
-1 & \\
& s_{2}
\end{array}\right)\left(\begin{array}{l}
\tilde{x} \\
\tilde{y}
\end{array}\right)+\left(\begin{array}{l}
f\left(u, v, \lambda^{*}\right) \\
g\left(u, v, \lambda^{*}\right)
\end{array}\right),
$$

where

$$
\begin{aligned}
& f\left(u, v, \lambda^{*}\right) \\
& \quad=\frac{a_{13}\left(s_{2}-a_{11}\right)-a_{12} a_{23}}{a_{12}\left(s_{2}+1\right)} u^{2}+\frac{a_{14}\left(s_{2}-a_{11}\right)-a_{12} a_{24}}{a_{12}\left(s_{2}+1\right)} u v
\end{aligned}
$$




$$
\begin{aligned}
& +\frac{a_{15}\left(s_{2}-a_{11}\right)-a_{12} a_{25}}{a_{12}\left(s_{2}+1\right)} v^{2}+\frac{b_{1}\left(s_{2}-a_{11}\right)-a_{12} c_{1}}{a_{12}\left(s_{2}+1\right)} u \lambda^{*} \\
& +\frac{b_{2}\left(s_{2}-a_{11}\right)-a_{12} c_{2}}{a_{12}\left(s_{2}+1\right)} v \lambda^{*}+\frac{b_{3}\left(s_{2}-a_{11}\right)-a_{12} c_{3}}{a_{12}\left(s_{2}+1\right)} u^{2} \lambda^{*} \\
& +\frac{b_{4}\left(s_{2}-a_{11}\right)-a_{12} c_{4}}{a_{12}\left(s_{2}+1\right)} u v \lambda^{*}+\frac{b_{5}\left(s_{2}-a_{11}\right)-a_{12} c_{5}}{a_{12}\left(s_{2}+1\right)} v^{2} \lambda^{*} \\
& +\frac{e_{1}\left(s_{2}-a_{11}\right)-a_{12} d_{1}}{a_{12}\left(s_{2}+1\right)} u^{3}+\frac{e_{2}\left(s_{2}-a_{11}\right)-a_{12} d_{2}}{a_{12}\left(s_{2}+1\right)} u^{2} v \\
& +\frac{e_{3}\left(s_{2}-a_{11}\right)-a_{12} d_{3}}{a_{12}\left(s_{2}+1\right)} v^{2} u+\frac{e_{4}\left(s_{2}-a_{11}\right)-a_{12} d_{4}}{a_{12}\left(s_{2}+1\right)} v^{3} \\
& +O\left(\left(|u|+|v|+\left|\lambda^{*}\right|\right)^{4}\right) \\
& g\left(u, v, \lambda^{*}\right) \\
& =\frac{a_{13}\left(1+a_{11}\right)+a_{12} a_{23}}{a_{12}\left(s_{2}+1\right)} u^{2}+\frac{a_{14}\left(1+a_{11}\right)+a_{12} a_{24}}{a_{12}\left(s_{2}+1\right)} u v \\
& +\frac{a_{15}\left(1+a_{11}\right)+a_{12} a_{25}}{a_{12}\left(s_{2}+1\right)} v^{2}+\frac{b_{1}\left(1+a_{11}\right)+a_{12} c_{1}}{a_{12}\left(s_{2}+1\right)} u \lambda^{*} \\
& +\frac{b_{2}\left(1+a_{11}\right)+a_{12} c_{2}}{a_{12}\left(s_{2}+1\right)} \nu \lambda^{*}+\frac{b_{3}\left(1+a_{11}\right)+a_{12} c_{3}}{a_{12}\left(s_{2}+1\right)} u^{2} \lambda^{*} \\
& +\frac{b_{4}\left(1+a_{11}\right)+a_{12} c_{4}}{a_{12}\left(s_{2}+1\right)} u v \lambda^{*}+\frac{b_{5}\left(1+a_{11}\right)+a_{12} c_{5}}{a_{12}\left(s_{2}+1\right)} v^{2} \lambda^{*} \\
& +\frac{e_{1}\left(1+a_{11}\right)+a_{12} d_{1}}{a_{12}\left(s_{2}+1\right)} u^{3}+\frac{e_{2}\left(1+a_{11}\right)+a_{12} d_{2}}{a_{12}\left(s_{2}+1\right)} u^{2} v \\
& +\frac{e_{3}\left(1+a_{11}\right)+a_{12} d_{3}}{a_{12}\left(s_{2}+1\right)} v^{2} u+\frac{e_{4}\left(1+a_{11}\right)+a_{12} d_{4}}{a_{12}\left(s_{2}+1\right)} v^{3} \\
& +O\left(\left(|u|+|v|+\left|\lambda^{*}\right|\right)^{4}\right)
\end{aligned}
$$

and

$$
\begin{aligned}
& u=a_{12}(\tilde{x}+\tilde{y}), \quad v=-\left(1+a_{11}\right) \tilde{x}+\left(s_{2}-a_{11}\right) \tilde{y}, \\
& u v=a_{12}\left[-\left(1+a_{11}\right) \tilde{x}^{2}+\left(s_{2}-2 a_{11}-1\right) \tilde{x} \tilde{y}+a_{12}\left(s_{2}-a_{11}\right) \tilde{y}^{2},\right. \\
& u^{2}=a_{12}^{2}(\tilde{x}+\tilde{y})^{2}, \quad v^{2}=\left[-\left(1+a_{11}\right) \tilde{x}+\left(s_{2}-a_{11}\right) \tilde{y}\right]^{2}, \\
& u v^{2}=a_{12}(\tilde{x}+\tilde{y})\left[-\left(1+a_{11}\right) \tilde{x}+\left(s_{2}-a_{11}\right) \tilde{y}\right]^{2}, \\
& u^{3}=a_{12}^{3}(\tilde{x}+\tilde{y})^{3}, \quad u^{2} v=a_{12}^{2}(\tilde{x}+\tilde{y})^{2}\left[-\left(1+a_{11}\right) \tilde{x}+\left(s_{2}-a_{11}\right) \tilde{y}\right], \\
& v^{3}=\left[-\left(1+a_{11}\right) \tilde{x}+\left(s_{2}-a_{11}\right) \tilde{y}\right]^{3} .
\end{aligned}
$$

There exists a center manifold $W_{c}(0,0,0)$ of Eq. (3.4) at the fixed point $(0,0)$ in a small neighborhood of $\lambda^{*}$. From the center manifold theorem, we know that there exists a center manifold

$$
\begin{aligned}
& W_{c}(0,0,0) \\
& \quad=\left\{\left(\tilde{x}, \tilde{y}, \lambda^{*}\right) \in R^{3}, \tilde{y}=h^{*}\left(\tilde{x}, \lambda^{*}\right)=a_{1} \tilde{x}^{2}+a_{2} \tilde{x} \lambda^{*}+a_{3} \lambda^{* 2}+O\left(\left(|\tilde{x}|+\left|\lambda^{*}\right|\right)^{3}\right)\right\}
\end{aligned}
$$


for $\tilde{x}$ and $\lambda^{*}$ sufficiently small. Then the center manifold must satisfy

$$
\begin{aligned}
& N\left(h^{*}\left(\tilde{x}, \lambda^{*}\right)\right) \\
& \quad=h^{*}\left(-\tilde{x}+f\left(\tilde{x}, h^{*}\left(\tilde{x}, \lambda^{*}\right), \lambda^{*}\right), \lambda^{*}\right)-s_{2} h^{*}\left(\tilde{x}, \lambda^{*}\right)-g\left(\tilde{x}, h^{*}\left(\tilde{x}, \lambda^{*}\right), \lambda^{*}\right)=0 .
\end{aligned}
$$

Substituting (3.4) and (3.5) into (3.6) and comparing coefficients of (3.6), we obtain where $O\left(\left(|\tilde{x}|+\left|\lambda^{*}\right|\right)^{3}\right)$ is a function in $\left(\tilde{x}, \lambda^{*}\right)$ at least of the third order, and

$$
\begin{aligned}
a_{1}= & -\frac{\left(1+a_{11}\right)\left[a_{14}\left(1+a_{11}\right)+a_{12} a_{24}\right]}{1-s_{2}^{2}}+\frac{a_{12}\left[\left(1+a_{11}\right) a_{13}+a_{12} a_{23}\right]}{1-s_{2}^{2}} \\
& +\frac{\left[a_{15}\left(1+a_{11}\right)+a_{12} a_{25}\right]\left(1+a_{11}\right)^{2}}{a_{12}\left(1-s_{2}^{2}\right)}, \\
a_{2}= & \frac{\left(1+a_{11}\right)\left[b_{2}\left(1+a_{11}\right)+a_{12} c_{2}\right]}{a_{12}\left(1+s_{2}\right)^{2}}-\frac{b_{1}\left(1+a_{11}\right)+a_{12} c_{1}}{\left(1+s_{2}\right)^{2}}, \quad a_{3}=0 .
\end{aligned}
$$

Therefore, model (1.2) restricted to the center manifold is given by

$$
F: \tilde{x} \rightarrow-\tilde{x}+h_{1} \tilde{x}^{2}+h_{2} \tilde{x} \lambda^{*}+h_{3} \tilde{x}^{2} \lambda^{*}+h_{4} \tilde{x} \lambda^{* 2}+h_{5} \tilde{x}^{3}+O\left(\left(|\tilde{x}|+\left|\lambda^{*}\right|\right)^{4}\right),
$$

where

$$
\begin{aligned}
h_{1}= & \frac{1}{a_{12}\left(s_{2}+1\right)}\left\{a_{12}^{2}\left[a_{13}\left(s_{2}-a_{11}\right)-a_{12} a_{23}\right]-a_{12}\left(1+a_{11}\right)\left[a_{14}\left(s_{2}-a_{11}\right)-a_{12} a_{24}\right]\right. \\
& \left.+\left[a_{15}\left(s_{2}-a_{11}\right)-a_{12} a_{25}\right]\left(1+a_{11}\right)^{2}\right\}, \\
h_{2}= & \frac{1}{a_{12}\left(1+s_{2}\right)}\left[\left[a_{12} b_{1}\left(s_{2}-a_{11}\right)-a_{12}^{2} c_{1}\right]-\left(1+a_{11}\right)\left[b_{2}\left(s_{2}-a_{11}\right)-a_{12} c_{2}\right]\right], \\
h_{3}= & \frac{a_{2}}{a_{12}\left(s_{2}+1\right)}\left\{2 a_{12}^{2}\left[a_{13}\left(s_{2}-a_{11}\right)-a_{12} a_{23}\right]\right. \\
& +a_{12}\left(s_{2}-1-2 a_{11}\right)\left[a_{14}\left(s_{2}-a_{11}\right)-a_{12} a_{24}\right] \\
& \left.-2\left(1+a_{11}\right)\left(s_{2}-a_{11}\right)\left[a_{15}\left(s_{2}-a_{11}\right)-a_{12} a_{25}\right]\right\} \\
& +\frac{a_{1}}{a_{12}\left(s_{2}+1\right)}\left\{\left[a_{12} b_{1}\left(s_{2}-a_{11}\right)-a_{12}^{2} c_{1}\right]\right. \\
& \left.+\left(s_{2}-a_{11}\right)\left[b_{2}\left(s_{2}-a_{11}\right)-a_{12} c_{2}\right]\right\}+\frac{1}{a_{12}\left(s_{2}+1\right)}\left\{a_{12}^{2}\left[b_{3}\left(s_{2}-a_{11}\right)-a_{12} c_{3}\right]\right. \\
& \left.-a_{12}\left(1+a_{11}\right)\left[b_{4}\left(s_{2}-a_{11}\right)-a_{12} c_{4}\right]+\left[b_{5}\left(s_{2}-a_{11}\right)-a_{12} c_{5}\right]\left(1+a_{11}\right)^{2}\right\}, \\
h_{4}= & \frac{a_{2}}{a_{12}\left(1+s_{2}\right)}\left\{\left[a_{12} b_{1}\left(s_{2}-a_{11}\right)-a_{12}^{2} c_{1}\right]+\left(s_{2}-a_{11}\right)\left[b_{2}\left(s_{2}-a_{11}\right)-a_{12} c_{2}\right]\right\}, \\
h_{5}= & \frac{1}{a_{12}\left(s_{2}+1\right)}\left\{2 a_{12}^{2} a_{1}\left[a_{13}\left(s_{2}-a_{11}\right)-a_{12} a_{23}\right]\right. \\
& +a_{1} a_{12}\left(s_{2}-1-2 a_{11}\right)\left[a_{14}\left(s_{2}-a_{11}\right)-a_{12} a_{24}\right] \\
& +a_{12}^{3}\left[e_{1}\left(s_{2}-a_{11}\right)-a_{12} d_{1}\right]-a_{12}^{2}\left(1+a_{11}\right)\left[e_{2}\left(s_{2}-a_{11}\right)-a_{12} d_{2}\right] \\
& -\left[e_{4}\left(s_{2}-a_{11}\right)-a_{12} d_{4}\right]\left(1+a_{11}\right)^{3} \\
& \left.+\left[e_{3}\left(s_{2}-a_{11}\right)-a_{12} d_{3}\right]\left(1+a_{11}\right)^{2}-2 a_{1}\left(1+a_{11}\right)\left(s_{2}-a_{11}\right)\left[a_{15}\left(s_{2}-a_{11}\right)-a_{12} a_{25}\right]\right\} .
\end{aligned}
$$



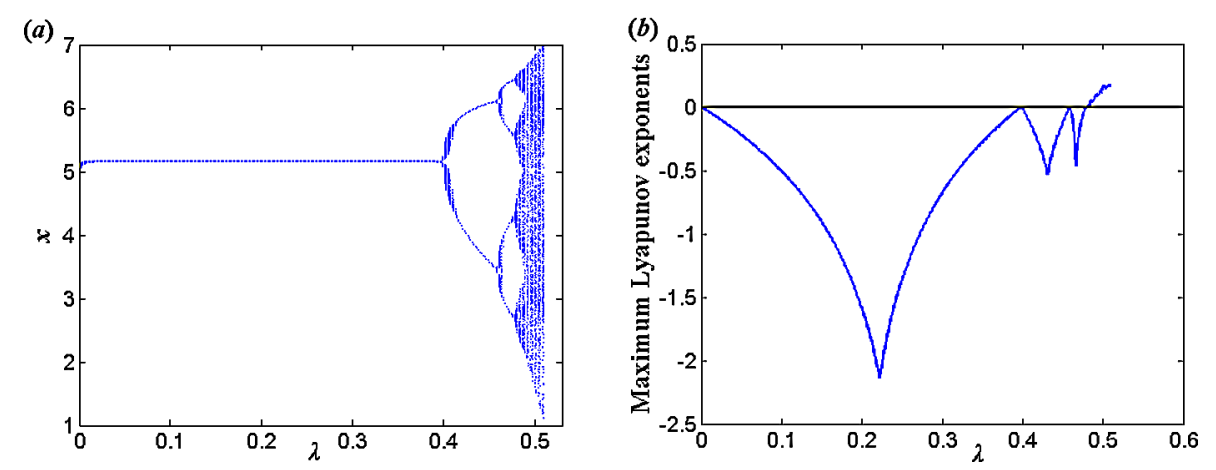

Figure 1 (a) Bifurcation diagram of map (1.2) with $\lambda \in(0,0.55), B=10, k=0.95, \beta=0.2$, the initial value is $(5,5)$; (b) Maximum Lyapunov exponents corresponding to $(\mathbf{a})$.

In order for map (3.7) to undergo a flip bifurcation, we require that two discriminatory quantities $\alpha_{1}$ and $\alpha_{2}$ are not zero, where

$$
\alpha_{1}=\left(\frac{\partial^{2} F}{\partial \tilde{x} \partial \lambda^{*}}+\frac{1}{2} \frac{\delta F}{\delta \lambda^{*}} \frac{\delta^{2} F}{\delta \tilde{x}^{2}}\right)_{(0,0)}=h_{2}
$$

and

$$
\alpha_{2}=\left(\frac{1}{6} \frac{\partial^{3} F}{\partial \tilde{x}^{3}}+\left(\frac{1}{2} \frac{\delta^{2} F}{\delta \tilde{x}^{2}}\right)^{2}\right)_{(0,0)}=h_{5}+h_{1}^{2}
$$

From the above analysis and the theorem of [23], we have the following result.

Theorem 3.1 If $\alpha_{2} \neq 0$, then map (1.2) undergoes a flip bifurcation at the fixed point $\left(x^{*}, y^{*}\right)$ when the parameter $\lambda$ varies in a small neighborhood of $\lambda_{1}$. Moreover, if $\alpha_{2}>0$ (resp., $\left.\alpha_{2}<0\right)$, then the period- 2 orbits that bifurcate from $\left(x^{*}, y^{*}\right)$ are stable (resp., unstable).

In Sect. 4 we will give some values of parameters such that $\alpha_{2} \neq 0$; thus, the flip bifurcation occurs as $\lambda$ varies (see Fig. 1).

\subsection{Neimark-Sacker bifurcation analysis}

Finally, we discuss the Neimark-Sacker bifurcation of $E\left(x^{*}, y^{*}\right)$ if parameters $\left(B, k, \beta, \lambda_{2}\right)$ vary in a small neighborhood of $\mathrm{HB} H_{B}$.

Taking parameters $\left(B, k, \beta, \lambda_{2}\right)$ arbitrarily from $H_{B}$, we consider system (1.2) with $\left(B, k, \beta, \lambda_{1}\right)$, which is described by

$$
\left\{\begin{array}{l}
x^{\prime} \rightarrow x+\lambda_{2} x\left(B \frac{x^{\beta}}{x^{\beta}+k y^{\beta}}-x\right), \\
y^{\prime} \rightarrow y+\lambda_{2} y\left(B \frac{k y^{\beta}}{x^{\beta}+k y^{\beta}}-y\right) .
\end{array}\right.
$$

Map (3.8) has a unique positive fixed point $E\left(x^{*}, y^{*}\right)$.

Choosing $\lambda^{*}$ as a bifurcation parameter, we consider a perturbation of (3.8) as follows:

$$
\left\{\begin{array}{l}
x^{\prime} \rightarrow x+\left(\lambda_{2}+\lambda^{*}\right) x\left(B \frac{x^{\beta}}{x^{\beta}+k y^{\beta}}-x\right), \\
y^{\prime} \rightarrow y+\left(\lambda_{2}+\lambda^{*}\right) y\left(B \frac{k y^{\beta}}{x^{\beta}+k y^{\beta}}-y\right),
\end{array}\right.
$$

where $\left|\lambda^{*}\right| \ll 1$, which is a small perturbation parameter. 
Let $u=x-x^{*}$ and $v=y-y^{*}$. Then we transform the fixed point $E\left(x^{*}, y^{*}\right)$ of map (3.9) into the origin. We have

$$
\begin{aligned}
& \left(\begin{array}{l}
u \\
v
\end{array}\right) \\
& \rightarrow\left(\begin{array}{c}
a_{11} u+a_{12} v+a_{13} u^{2}+a_{14} u v+a_{15} v^{2}+e_{1} u^{3}+e_{2} u^{2} v+e_{3} v^{2} u+e_{4} v^{3}+O\left((|u|+|v|)^{4}\right) \\
a_{21} u+a_{22} v+a_{23} u^{2}+a_{24} u v+a_{25} v^{2}+d_{1} u^{3}+d_{2} u^{2} v+d_{3} v^{2} u+d_{4} v^{3}+O\left((|u|+|v|)^{4}\right)
\end{array}\right),
\end{aligned}
$$

where $a_{11}, a_{12}, a_{13}, a_{14}, a_{15}, e_{1}, e_{2}, e_{3}, e_{4}, a_{21}, a_{22}, a_{23}, a_{24}, a_{25}, d_{1}, d_{2}, d_{3}, d_{4}$ are given in (3.3) by substituting $\lambda$ for $\lambda_{2}+\lambda^{*}$.

Note that the characteristic equation associated with the linearization of map (3.10) at $(u, v)=(0,0)$ is given by

$$
s^{2}+P\left(\bar{\lambda}^{*}\right) s+Q\left(\bar{\lambda}^{*}\right)=0
$$

where

$$
\begin{aligned}
& P\left(\bar{\lambda}^{*}\right)=-2-G\left(\lambda_{2}+\bar{\lambda}^{*}\right), \\
& Q\left(\bar{\lambda}^{*}\right)=1+G\left(\lambda_{2}+\bar{\lambda}^{*}\right)+H\left(\lambda_{2}+\bar{\lambda}^{*}\right)^{2} .
\end{aligned}
$$

Since parameters $\left(B, k, \beta, \lambda_{2}\right) \in H_{B}$, the eigenvalues of $(0,0)$ are a pair of complex conjugate numbers $s$, and $\bar{s}$ with modulus one by Proposition 4 , where

$$
s, \bar{s}=-\frac{P\left(\bar{\lambda}^{*}\right)}{2} \pm \frac{i}{2} \sqrt{4 Q\left(\bar{\lambda}^{*}\right)-P^{2}\left(\bar{\lambda}^{*}\right)}=1+\frac{\left(\lambda_{2}+\lambda^{*}\right)}{2} \pm \frac{i\left(\lambda_{2}+\lambda^{*}\right)}{2} \sqrt{4 H-G^{2}}
$$

Moreover, we have

$$
|s|_{\bar{\lambda}^{*}=0}=\sqrt{Q(0)}=1, \quad l=\left.\frac{d|s|}{d \bar{\lambda}^{*}}\right|_{\bar{\lambda}^{*}=0}=-\frac{G}{2} \neq 0 .
$$

Also, it requires that when $\bar{\lambda}^{*}=0, \lambda^{m}, \bar{\lambda}^{m} \neq 1(m=1,2,3,4)$ which is equivalent to $P(0) \neq$ $-2,0,1,2$. Note that $\left(B, k, \beta, \lambda_{2}\right) \in H_{B}$. Thus, $P(0) \neq-2,2$. We only need to require that $P(0) \neq 0,1$, which leads to

$$
G^{2} \neq 2 H, 3 H
$$

Therefore, the eigenvalues $s, \bar{s}$ of a fixed point $(0,0)$ of $(3.10)$ do not lie in the intersection of the unit circle with the coordinate axes when $\delta$ and (3.11) holds.

Next, we study the normal form of (3.10) at $\bar{\lambda}^{*}=0$.

$$
\text { Let } \begin{array}{r}
\bar{\lambda}^{*}=0, \mu=1+\frac{G \lambda_{2}}{2}, \omega \\
T=\left(\begin{array}{cc}
a_{12} & 0 \\
\mu-a_{11} & -\omega
\end{array}\right) .
\end{array}
$$

Moreover, using the translation

$$
\left(\begin{array}{l}
u \\
v
\end{array}\right)=T\left(\begin{array}{l}
\tilde{x} \\
\tilde{y}
\end{array}\right)
$$


then system (3.10) becomes

$$
\left(\begin{array}{c}
\tilde{x} \\
\tilde{y}
\end{array}\right) \rightarrow\left(\begin{array}{cc}
\mu & -\omega \\
\omega & \mu
\end{array}\right)\left(\begin{array}{c}
\tilde{x} \\
\tilde{y}
\end{array}\right)+\left(\begin{array}{c}
\tilde{f}(\tilde{x}, \tilde{y}) \\
\tilde{g}(\tilde{x}, \tilde{y})
\end{array}\right),
$$

where

$$
\begin{aligned}
& \tilde{f}(\tilde{x}, \tilde{y})= \frac{a_{13}}{a_{12}} u^{2}+\frac{a_{14}}{a_{12}} u v+\frac{a_{15}}{a_{12}} v^{2}+\frac{e_{1}}{a_{12}} u^{3}+\frac{e_{2}}{a_{12}} u^{2} v+\frac{e_{3}}{a_{12}} v^{2} u+\frac{e_{4}}{a_{12}} v^{3}+O\left((|\tilde{x}|+|\tilde{y}|)^{4}\right), \\
& \tilde{g}(\tilde{x}, \tilde{y})= \frac{a_{13}\left(\mu-a_{11}\right)-a_{12} a_{23}}{a_{12} \omega} u^{2}+\frac{a_{14}\left(\mu-a_{11}\right)-a_{12} a_{24}}{a_{12} \omega} u v \\
&+\frac{a_{15}\left(\mu-a_{11}\right)-a_{12} a_{25}}{a_{12} \omega} v^{2}+\frac{e_{1}\left(\mu-a_{11}\right)-a_{12} d_{1}}{a_{12} \omega} u^{3} \\
&+\frac{e_{2}\left(\mu-a_{11}\right)-a_{12} d_{2}}{a_{12} \omega} u^{2} v+\frac{e_{3}\left(\mu-a_{11}\right)-a_{12} d_{3}}{a_{12} \omega} v^{2} u \\
&+\frac{e_{4}\left(\mu-a_{11}\right)-a_{12} d_{4}}{a_{12} \omega} v^{3}+O\left((|\tilde{x}|+|\tilde{y}|)^{4}\right), \\
& u^{2}= a_{12}^{2} \tilde{x}^{2}, \quad u v=a_{12}\left(\mu-a_{11}\right) \tilde{x}^{2}-a_{12} \omega \tilde{x} \tilde{y}, \\
& v^{2}=\left(\mu-a_{11}\right)^{2} \tilde{x}^{2}-2\left(\mu-a_{11}\right) \omega \tilde{x} \tilde{y}+\omega^{2} \tilde{y}^{2} \\
& v^{2} u= \\
& u_{12}\left(\mu-a_{11}\right)^{2} \tilde{x}^{3}-2 a_{12}\left(\mu-a_{11}\right) \omega \tilde{x}^{2} \tilde{y}+a_{12} \omega^{2} \tilde{x}^{2} \tilde{y}^{2}, \\
& u^{2} v=a_{12}^{2}\left(\mu-a_{11}\right) \tilde{x}^{3}-a_{12}^{2} \omega \tilde{x}^{2} \tilde{y} \\
& u^{3}=a_{12}^{3} \tilde{x}^{3}, \quad v^{3}=\left(\mu-a_{11}\right)^{3} \tilde{x}^{3}-3 \omega\left(\mu-a_{11}\right)^{2} \tilde{x}^{2} \tilde{y}+3 \omega^{2}\left(\mu-a_{11}\right) \tilde{x} \tilde{y}^{2}-\omega^{3} \tilde{y}^{3} .
\end{aligned}
$$

Therefore,

$$
\begin{aligned}
\tilde{f}_{\tilde{x} \tilde{x}}= & 2 a_{12} a_{13}+2 a_{14}\left(\mu-a_{11}\right)+\frac{2 a_{15}}{a_{12}}\left(\mu-a_{11}\right)^{2}, \quad \tilde{f}_{\tilde{x} \tilde{y}}=-a_{14} \omega-\frac{2 a_{15}}{a_{12}}\left(\mu-a_{11}\right) \omega \\
\tilde{f}_{\tilde{y} \tilde{y}}= & \frac{a_{15}}{a_{12}} \omega^{2}, \\
\tilde{f}_{\tilde{x} \tilde{x} \tilde{x}}= & 6 e_{1} a_{12}^{2}+6 e_{2} a_{12}\left(\mu-a_{11}\right)+6 e_{3}\left(\mu-a_{11}\right)^{2}+\frac{6 e_{4}}{a_{12}}\left(\mu-a_{11}\right)^{3}, \quad \tilde{f}_{\tilde{y} \tilde{y} \tilde{y}}=-\frac{e_{4}}{a_{12}} \omega^{2}, \\
\tilde{f}_{\tilde{x} \tilde{y}}= & \frac{6}{a_{12}} \omega^{2} e_{4}\left(\mu-a_{11}\right), \\
\tilde{f}_{\tilde{x} \tilde{x} \tilde{y}}= & -4 \omega e_{3}\left(\mu-a_{11}\right)-2 \omega e_{2} a_{12}-\frac{6}{a_{12}}\left(e_{4} \omega\left(\mu-a_{11}\right)^{2}\right), \\
\tilde{g}_{\tilde{x} \tilde{x}}= & \frac{2}{\omega}\left\{a_{12}\left[a_{13}\left(\mu-a_{11}\right)-a_{12} a_{23}\right]+\left(\mu-a_{11}\right)\left[a_{14}\left(\mu-a_{11}\right)-a_{12} a_{24}\right]\right. \\
& \left.+\left[a_{15}\left(\mu-a_{11}\right)-a_{12} a_{25}\right]\left(\mu-a_{11}\right)^{2}\right\}, \\
\tilde{g}_{\tilde{x} \tilde{y}}= & \frac{1}{a_{12}}\left\{a_{12}\left[a_{12} a_{24}-a_{14}\left(\mu-a_{11}\right)\right]-2\left(\mu-a_{11}\right)\left[a_{15}\left(\mu-a_{11}\right)-a_{12} a_{25}\right]\right\} \\
\tilde{g}_{\tilde{y} \tilde{y}=}= & \frac{2 \omega}{a_{12}}\left[a_{15}\left(\mu-a_{11}\right)-a_{12} a_{25}\right], \\
\tilde{g}_{\tilde{x} \tilde{x} \tilde{x}}= & \frac{6}{\omega}\left\{a_{12}^{2}\left[e_{1}\left(\mu-a_{11}\right)-a_{12} d_{1}\right]+a_{12}\left(\mu-a_{11}\right)\left[e_{3}\left(\mu-a_{11}\right)-a_{12} d_{3}\right]\right. \\
& +\left[e_{3}\left(\mu-a_{11}\right)-a_{12} d_{3}\right]\left(\mu-a_{11}\right)^{2}
\end{aligned}
$$




$$
\begin{aligned}
& \left.+\left[e_{4}\left(\mu-a_{11}\right)-a_{12} d_{4}\right]\left(\mu-a_{11}\right)^{3}\right\}, \\
\tilde{g}_{\tilde{y} \tilde{y} \tilde{y}}= & \frac{6 \omega^{2}}{a_{12}}\left[a_{12} d_{4}-e_{4}\left(\mu-a_{11}\right)\right], \\
\tilde{g}_{\tilde{x} \tilde{y} \tilde{y}}= & \frac{6}{a_{12}} \omega\left(\mu-a_{11}\right)\left[e_{4}\left(\mu-a_{11}\right)-a_{12} d_{4}\right], \\
\tilde{g}_{\tilde{x} \tilde{x} \tilde{y}}= & 4\left(\mu-a_{11}\right)\left[a_{12} d_{3}-e_{3}\left(\mu-a_{11}\right)\right]+2 a_{12}\left[a_{12} d_{2}-e_{2}\left(\mu-a_{11}\right)\right] \\
& +\frac{6}{a_{12}}\left[a_{12} d_{4}-e_{4}\left(\mu-a_{11}\right)\right]\left(\mu-a_{11}\right)^{2}
\end{aligned}
$$

at point $(0,0)$.

In order for system (3.12) to undergo the Neimark-Sacker bifurcation, we require that the following discriminatory quantity is not zero:

$$
a=\left.\left\{-\operatorname{Re}\left(\frac{(1-2 s) \bar{s}^{2}}{1-s} \xi_{20} \xi_{11}\right)-\frac{1}{2}\left|\xi_{11}\right|^{2}-\left|\xi_{02}\right|^{2}+\operatorname{Re}\left(\bar{s} \xi_{21}\right)\right\}\right|_{\bar{\lambda}^{*}=0}
$$

where

$$
\begin{aligned}
& \xi_{20}=\frac{1}{8}\left[\left(\tilde{f}_{\tilde{x} \tilde{x}}-\tilde{f}_{\tilde{y} \tilde{y}}+2 \tilde{g}_{\tilde{x} \tilde{y}}\right)+i\left(\tilde{g}_{\tilde{x} \tilde{x}}-\tilde{g}_{\tilde{y} \tilde{y}}-2 \tilde{f}_{\tilde{x} \tilde{y}}\right)\right], \\
& \xi_{11}=\frac{1}{4}\left[\left(\tilde{f}_{\tilde{x} \tilde{x}}+\tilde{f}_{\tilde{y} \tilde{y}}\right)+i\left(\tilde{g}_{\tilde{x} \tilde{x}}+\tilde{g}_{\tilde{y} \tilde{y}}\right)\right], \\
& \xi_{02}=\frac{1}{8}\left[\left(\tilde{f}_{\tilde{x} \tilde{x}}-\tilde{f}_{\tilde{y} \tilde{y}}-2 \tilde{g}_{\tilde{x} \tilde{y}}\right)+i\left(\tilde{g}_{\tilde{x} \tilde{x}}-\tilde{g}_{\tilde{y} \tilde{y}}+2 \tilde{f}_{\tilde{x} \tilde{y}}\right)\right], \\
& \xi_{21}=\frac{1}{16}\left[\left(\tilde{f}_{\tilde{x} \tilde{x} \tilde{x}}+\tilde{f}_{\tilde{x} \tilde{y} \tilde{y}}+\tilde{g}_{\tilde{x} \tilde{x} \tilde{y}}+\tilde{g}_{\tilde{y} \tilde{y} \tilde{y}}\right)+i\left(\tilde{g}_{\tilde{x} \tilde{x} \tilde{x}}+\tilde{g}_{\tilde{x} \tilde{y} \tilde{y}}-\tilde{f}_{\tilde{x} \tilde{x} \tilde{y}}-\tilde{f}_{\tilde{y} \tilde{y} \tilde{y}}\right)\right] .
\end{aligned}
$$

From the above analysis, we have the following theorem.

Theorem 3.2 If condition (3.11) holds and $a \neq 0$, then map (1.2) undergoes the NeimarkSacker bifurcation at the fixed point $\left(x^{*}, y^{*}\right)$ when the parameter $\lambda$ varies in a small neighborhood of $\lambda_{2}$. Moreover, if $a<0$ (resp., $a>0$ ), then an attracting (resp., repelling) invariant closed curve bifurcates from the fixed point for $\lambda>\lambda_{2}$ (resp., $\left.\lambda<\lambda_{2}\right)$.

\section{Numerical simulations}

In this section, we illustrate the above analytic results and show the complex dynamical behaviors by the bifurcation diagrams, phase portraits, and maximum Lyapunov exponents for system (1.2).

For the sake of analysis, we consider the symmetric case of identical firms, let $\lambda_{1}=\lambda_{2}=\lambda$, $\beta_{1}=\beta_{2}=\beta$. Then, from system (1.2), we obtain

$$
\left\{\begin{array}{l}
x^{\prime} \rightarrow x+\lambda x\left(B \frac{x^{\beta}}{x^{\beta}+k y^{\beta}}-x\right), \\
y^{\prime} \rightarrow y+\lambda y\left(B \frac{k y^{\beta}}{x^{\beta}+k y^{\beta}}-y\right) .
\end{array}\right.
$$

The bifurcation analyses are considered in the following cases:

(i) Varying $\lambda$ in range $0<\lambda<0.51$ and fixing $\beta=0.2, B=10, k=0.95$;

(ii) Varying $B$ in range $0<B<62.8$ and fixing $\beta=0.2, \lambda=0.08, k=0.95$; 
(iii) Varying $\beta$ in range $0.3<\beta<1$ and fixing $k=0.8, \lambda=0.4, B=10$;

(iv) Varying $k$ in range $0.35<k<2.5$ and fixing $\beta=0.2, \lambda=0.4, B=10$.

For case (i). The bifurcation diagram of system (1.2) in the $(\lambda, x)$ plane for $0<\lambda<0.51$ with initial values $\left(x_{0}, y_{0}\right)=(5,5)$ is given in Fig. 1 (a) to show the dynamical changes as $\lambda$ varies. The maximum Lyapunov exponents corresponding to the bifurcation diagram in Fig. 1(a) are given in Fig. 1(b).

In Fig. 1, we can see that there is a stable fixed point $(5.1602,4.8398)$ for $0<\lambda<0.3923$, and a flip bifurcation occurs at $\lambda=0.3923$. We observe that there are period- 2 orbits for larger regions $\lambda \in(0.3923,0.4571)$.

Figure 2 shows the phase portraits which are associated with Fig. 1 . For $\lambda \in(0,0.55)$, there are period-1,2,4 orbits (in Fig. 2 (a) (c)). Figure $2(\mathrm{~d})$ shows one of the stable fixed points. Figure 2(e) shows that the Hopf bifurcations emerge from the fixed points at $\lambda=$ 0.48 . When $\lambda=0.495$, we can see the chaotic sets in Fig. 2(f). The maximum Lyapunov
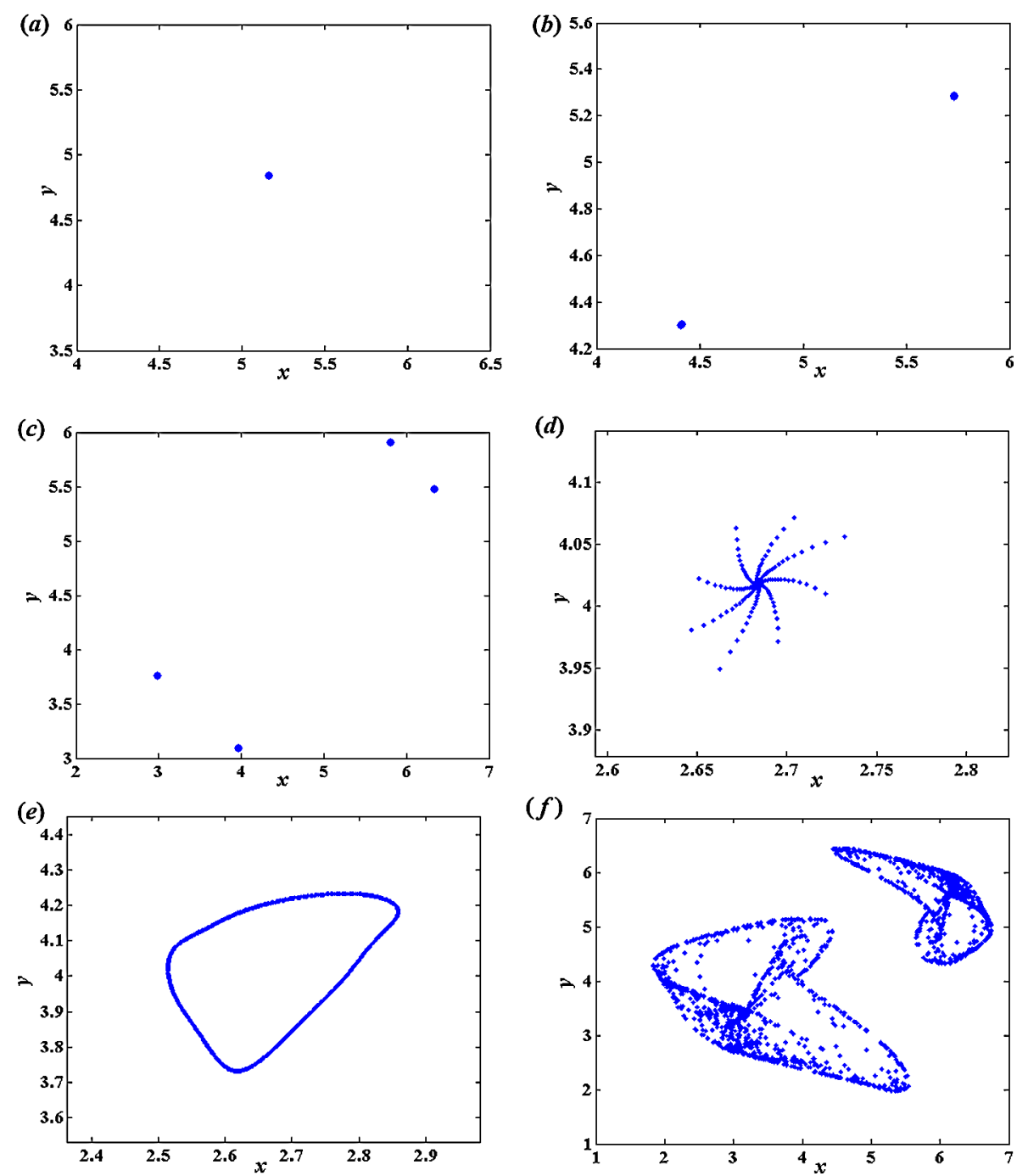

Figure 2 Phase plane for map (1.2) with $B=10, k=0.95, \beta=0.2$ and different $\lambda$. 

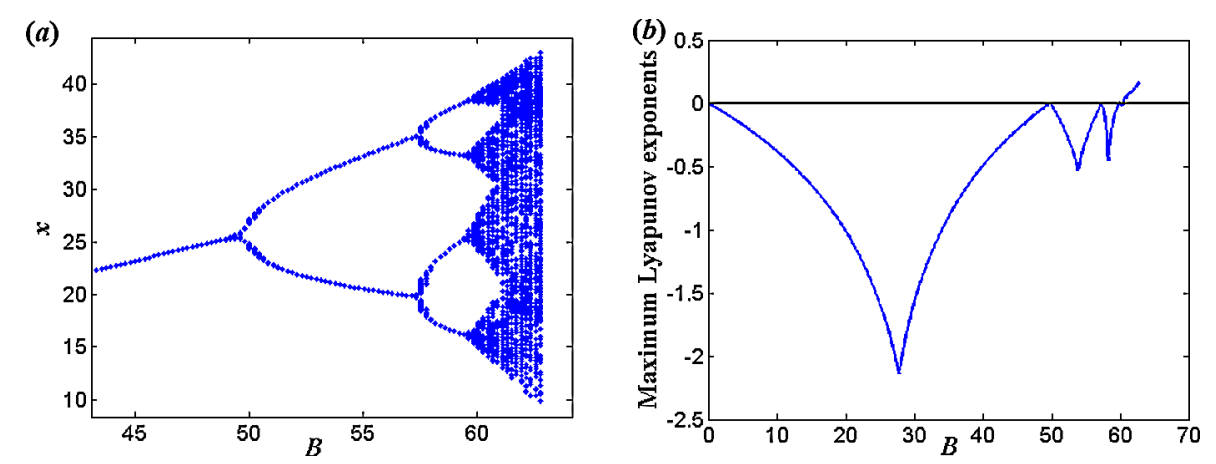

Figure 3 (a) Bifurcation diagram of map (1.2) with $B \in(0,62.8), \beta=0.2, k=0.95, \lambda=0.08$, the initial value is $(5,5)$; (b) Maximum Lyapunov exponents corresponding to (a).
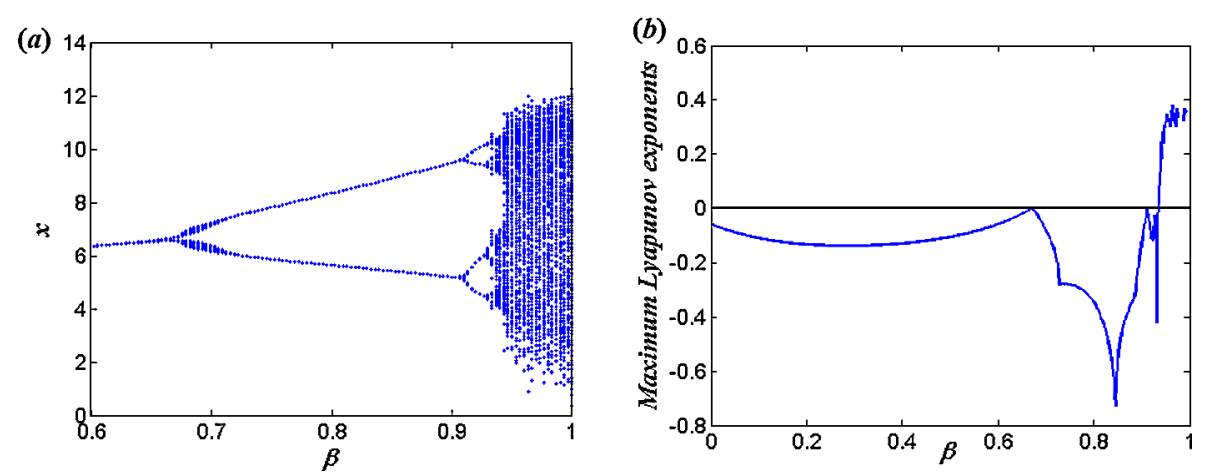

Figure 4 (a) Bifurcation diagram of map (1.2) with $\beta \in(0,1), k=0.8, B=10, \lambda=0.35$, the initial value is $(5,5)$; (b) Maximum Lyapunov exponents corresponding to (a).

exponents corresponding to $\lambda=0.495$ are larger than zero, which confirms the existence of the chaotic sets in Fig. 1(b).

For case (ii). The bifurcation diagram of system (1.2) in the $(B, x)$ plane for $0<B<62.8$ with initial values $\left(x_{0}, y_{0}\right)=(5,5)$ is given in Fig. 3(a) to show the dynamical changes as $B$ varies. The maximum Lyapunov exponents corresponding to the bifurcation diagram in Fig. 3(a) are given in Fig. 3(b).

In Fig. 3, a flip bifurcation occurs at $B=49.38$ by Proposition 1 . We observe that there are period-2 orbits for larger regions $B \in(49.38,57.35)$. Other cases are similar to case (i).

For case (iii). The bifurcation diagram of system (1.2) in the $(\beta, x)$ plane for $0<\beta<1$ with initial values $\left(x_{0}, y_{0}\right)=(5,5)$ is given in Fig. 3(a) to show the dynamical changes as $\beta$ varies. The maximum Lyapunov exponents corresponding to the bifurcation diagram in Fig. 4(a) are given in Fig. 4(b).

In Fig. 4, we can see that a flip bifurcation occurs at $\beta=0.6589$ by Proposition 1 . We observe that there are period-2 orbits for larger regions $\beta \in(0.6589,0.903)$. Other cases are similar to case (i).

For case (iv). The bifurcation diagram of system (1.2) in the $(k, x)$ plane for $0.355<k<2.5$ with initial values $\left(x_{0}, y_{0}\right)=(5,5)$ is given in Fig. 5(a) to show the dynamical changes as $k$ varies. The maximum Lyapunov exponents corresponding to the bifurcation diagram in Fig. 5(a) are given in Fig. 5(b). 
(a)

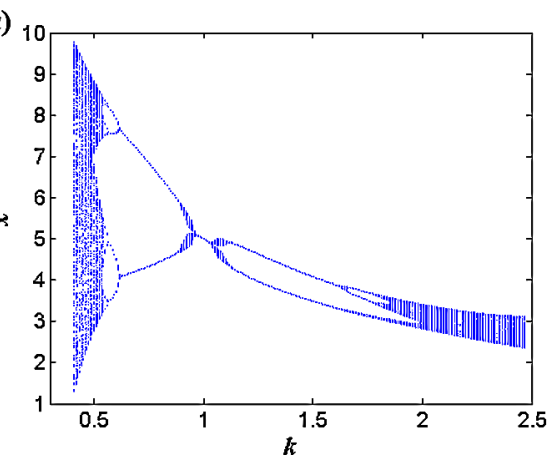

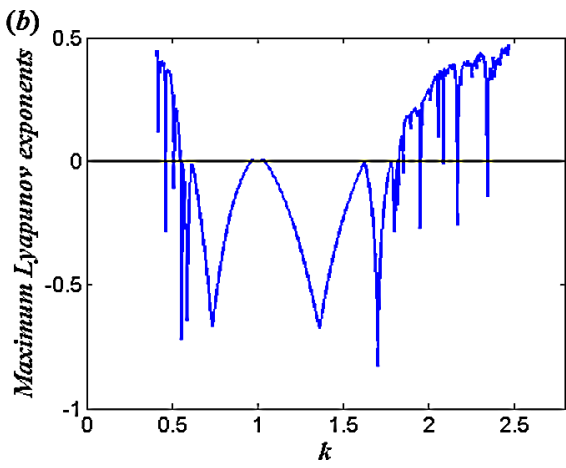

Figure 5 (a) Bifurcation diagram of map (1.2) with $k \in(0.35,2.5), \beta=0.2, B=10, \lambda=0.4$, the initial value is $(5,5)$; (b) Maximum Lyapunov exponents corresponding to $(\mathbf{a})$.

In Fig. 5, there exist double period doubling bifurcations and chaos. We can see that a flip bifurcation occurs at $k=0.9773$ or $k=1.027$ by Proposition 1 . We observe that there are period-2 orbits for regions $k \in(0.6261,0.9773)$ or $k \in(1.027,1.613)$. There are period-1, $2,4,6,8,16$ orbits with $k=1,0.7,0.58,1.95,0.56,0.55$. When $k=0.45,1.9,2.15$, we can see the chaotic sets in Fig. 5(a). The maximum Lyapunov exponents corresponding to them are greater than zero, which implies the existence of the chaotic sets in Fig. 5(b).

\section{Discussion}

In this paper, we discuss the dynamical behaviors of model (1.2). From the discussion in Sect. 2, we know that there exist flip bifurcation and chaos about equilibrium as the parameters vary in the small neighborhood. We have obtained a global qualitative analysis of model (1.2) depending on all parameters and showed that the model exhibits the bifurcations. By choosing $\lambda, B, \beta, k$ as bifurcation parameters, respectively, it was shown that the model undergoes a series of bifurcations including the flip bifurcation, period doubling bifurcation, and chaos. Moreover, system (1.2) exhibits many complex dynamic behaviors, including period-1, 2, 4, 6, 8, 16 orbits, invariant cycle, a cascade of period-doubling, quasi-periodic orbits, and the chaotic sets. These results reveal far richer dynamics of the discrete model compared to the continuous model.

Acknowledgements

The authors thank the anonymous reviewers for their valuable comments.

Funding

This work is supported by the National Natural Science Foundation of China under Grant 61472374, 71503096. China University of Geosciences (Wuhan) Graduate Quality Textbook Construction Project (YJC2017405).

\section{Competing interests}

The authors declare that they have no competing interests.

Authors' contributions

All authors read and approved the final manuscript.

Author details

${ }^{1}$ School of Education, Minzu University of China, Beijing, P.R. China. ${ }^{2}$ School of Automation, China University of Geosciences, Wuhan, P.R. China. ${ }^{3}$ Department of Mechanical Engineering, Boston University, Boston, USA.

Publisher's Note

Springer Nature remains neutral with regard to jurisdictional claims in published maps and institutional affiliations. 


\section{References}

1. Naert, P., Weverbergh, M.: On the prediction power of market share attraction models. J. Mark. Res. 18, 146-153 (1981)

2. Monahan, G.E., Sobel, M.J.: Stochastic dynamic market share attraction games. Games Econ. Behav. 6, 130-149 (1994)

3. Bischi, G., Gardini, L., Kopel, M.: Analysis of global bifurcations in a market share attraction model. J. Econ. Dyn. Control 24(5), 855-879 (2000)

4. Kopel, M., Bischi, G.l., Gardini, L.: On new phenomena in dynamic promotional competition models with homogeneous and quasi-homogeneous firms. In: Delli, D.G., Gallegati, M., Kirman, A.P. (eds.) Interaction and Market Structure. Essays on Heterogeneity in Economics, vol. 484, pp. 55-87. Springer, Berlin (2000)

5. Fok, D., Franses, P.H.: Analyzing the effects of a brand introduction on competitive structure using a market share attraction model. Int. J. Res. Mark. 21(2), 159-177 (2004)

6. Jing, Z., Jia, Z., Wang, R.: Chaos behavior in the discrete BVP oscillator. Int. J. Bifurc. Chaos 12, 619-627 (2002)

7. Jing, Z., Yang, J.P.: Bifurcation and chaos in discrete-time predator-prey system. Chaos Solitons Fractals 27, 259-277 (2006)

8. Liu, X., Xiao, D.: Complex dynamic behaviors of a discrete-time predator-prey system. Chaos Solitons Fractals 32 80-94 (2007)

9. Fan, D., Wei, J.: Bifurcation analysis of discrete survival red blood cells model. Commun. Nonlinear Sci. Numer. Simul. 14(8), 3358-3368 (2009)

10. Wang, B., Jian, J.: Stability and Hopf bifurcation analysis on a four-neuron BAM neural network with distributed delays Commun. Nonlinear Sci. Numer. Simul. 15, 189-204 (2010)

11. Shu, H., Wei, J.: Bifurcation analysis in a discrete BAM network model with delays. J. Differ. Equ. Appl. 17(1), 69-84 (2011)

12. Hu, Z.Y., Teng, Z., Zhang, L.: Stability and bifurcation analysis of a discrete predator-prey model with nonmonotonic functional response. Nonlinear Anal. 12, 2356-2377 (2011)

13. Jiang, X.W., Ding, L., Guan, Z.H., Yuan, F.S.: Bifurcation and chaotic behavior of a discrete-time Ricardo-Malthus model Nonlinear Dyn. 71, 437-446 (2013)

14. He, Z.M., Li, B.O.: Complex dynamic behavior of a discrete-time predator-prey system of Holling-III type. Adv. Differ. Equ. 2014, 180 (2014)

15. Xiao, M., Zheng, W., Cao, J.: Stability and bifurcation of genetic regulatory networks with small RNAs and multiple delays. Int. J. Comput. Math. 91, 5907-5927 (2014)

16. Ling, G., Guan, Z.H., Liao, R.: Stability and bifurcation analysis of cyclic genetic regulatory networks with mixed time delays. SIAM J. Appl. Dyn. Syst. 14(1), 202-220 (2015)

17. Cheng, L., Cao, H.: Bifurcation analysis of a discrete-time ratio-dependent predator-prey model with Allee effect. Commun. Nonlinear Sci. Numer. Simul. 38, 288-302 (2016)

18. Yu, P., Lin, W.: Complex dynamics in biological systems arising from multiple limit cycle bifurcation. J. Biol. Dyn. 10 , 263-285 (2016)

19. Abdelrahman, M.A.E., Chatzarakis, G.E., Li, T., Moaaz, O.: On the difference equation $x_{n+1}=a x_{n}+b x_{n-k}+f\left(x_{n-l} ; x_{n-k}\right)$. Adv. Differ. Equ. 2018, 431 (2018)

20. Wan, X., Wang, Z., Wu, M., Liu, X.: $H_{\infty}$ state estimation for discrete-time nonlinear singularly perturbed complex networks under the Round-Robin protocol. IEEE Trans. Neural Netw. Learn. Syst. PP(99), 1-12 (2018)

21. Albert, C.J.L.: Regularity and Complexity in Dynamical Systems. Springer, New York (2012)

22. Guckenheimer, J., Holmes, P.: Nonlinear Oscillations, Dynamical System and Bifurcation of Vector Fields. Springer, New York (1983)

23. Robinson, C.: Dynamical Systems, Stability, Symbolic Dynamics and Chaos, 2nd edn. CRC Press, Boca Raton (1999)

24. Kuznetsov, Y.A.: Elements of Applied Bifurcation Theory, Applied Mathematical Sciences, 3rd edn. Springer, New York (2004)

25. Wiggins, S.: Introduction to Applied Nonlinear Dynamical Systems and Chaos. Springer, New York (2003)

\section{Submit your manuscript to a SpringerOpen ${ }^{\circ}$ journal and benefit from:}

- Convenient online submission

- Rigorous peer review

- Open access: articles freely available online

- High visibility within the field

- Retaining the copyright to your article

Submit your next manuscript at $\boldsymbol{s p r i n g e r o p e n . c o m ~}$ 